\title{
GALACTIC BULGES AS AN EVOLUTIONARY PHENOMENON: OBSERVATIONAL EVIDENCE FROM THE STATISTICS OF "BOX/PEANUT" BULGES
}

\author{
R.-J. DETTMAR \\ Space Telescope Science Institute ${ }^{* \dagger}$ \\ 3700 San Martin Dr. \\ Baltimore, MD 21218 (USA)
}

\section{Introduction}

$\mathrm{N}$-body simulations for stars in bar potentials have demonstrated over the last couple of years (e.g., Combes et al. 1990; Raha et al. 1991) that bars growing in $\mathrm{z}$ will lead to the presence of a bulge component. In such a scenario a central "bulge" component could develop as a resonance phenomenon caused by a bar and would be distinctly different from any "spheroidal" or "halo" component.

There is growing evidence that our Galaxy harbors such a "bulge-bar" structure. The box- or peanut shape of the bulge in the Milky Way as revealed by the DIRBE experiment on board of COBE is one of the typical signatures for the proposed "resonant phenomenon". In addition to morphology gas kinematics shows the presence of a bar and the Galaxy and extragalactic examples have been discussed extensively earlier at this conference (see, e. g., contributions by Blitz, Gerhard, Combes, and Kuijken). Here we discuss evidence for such an evolutionary scenario in the formation of bulges based on the frequency of box- and peanut-shaped (b/p) bulges observed in a CCD surface photometry of edge-on galaxies.

*affiliated with the Astrophysics Division in the Space Science Department of ESA

${ }^{\dagger}$ present address: Astronomisches Institut der Ruhr-Universität, Universitätsstr. 150, D-44780 Bochum, Germany 


\section{Statistics of $b / p$ Bulges from CCD Photometry}

We have obtained CCD images of $\approx 80$ edge-on galaxies with the $2.2 \mathrm{~m}$ telescopes at ESO/La Silla and Calar Alto/Spain. Detailed surface photometry of a subset is presented in Barteldrees and Dettmar (1994).

As an example we show in Fig. 1 the surface photometry of ESO 321G10. This example demonstrates that the peanut shape of the bulge can be identified by the double humped profile in cuts taken parallel to the major axis. This is true even in spirals of types as late as Sc. In total 35 or $43 \%$ of the objects in our sample show b/p bulges. In Fig. 2 we have scaled our sample to the distribution of morphological types as given by the ESO/Uppsala catalogue. The particular binning of types is for easier comparison with previous work (Jarvis 1986, Shaw 1987, de Souza and dos Anjos 1987). While for types in the range S0 - Sbc the agreement with Shaw and de Souza \& dos Anjos is excellent, we find a significant fraction of later type spirals to show $b / p$ bulges. The previous studies used the sky survey photographic material and the higher dynamic range of our CCD photometry easily explains the higher detection rate in Sc galaxies.

It is worthwhile to point out that some more $b / p$ bulges in spirals of type Sbc-Sc can be found in the color images by Wray (1988). These images nicely represent the nuclear regions of galaxies and reveal well known objects such as NGC 5170 to have a b/p bulge.

The frequency of $b / p$ bulges now can to be compared with the frequency of bars broken down by morphological type. These numbers are very uncertain and $25 \%-35 \%$ seems to be a very conservative estimate for the fraction of barred galaxies (Sellwood and Wilkinson 1993) averaged over all spirals. With the large statistical errors from the small number of galaxies in our sample the frequencies seem to be compatible. However, the statistics of $b / p$ bulges is not corrected for the aspect angle. Therefore either bars have to be more frequent than $35 \%$ to explain $b / p$ bulges or other processes are contributing to the formation of $b / p$ bulges, too. In our sample there is indeed evidence that some $b / p$ bulges are caused by interaction and examples are presented elsewhere (Dettmar and Barteldrees 1990).

\section{Minor Axis Profiles}

The N-body simulations mentioned above (e.g., May et al. 1985; Combes et al. 1990) predict for the resulting "thick bar" surface brightness distributions that are significantly different from de Vaucouleurs profiles which well describe the spheroidal components in early type spiral galaxies. At least for some bulges of late type spirals the minor axis intensity profiles seem to fall of steeper than a de Vaucouleurs profile. For a large range in radius the de Vaucouleurs law is well represented by a simple $\mathrm{r}^{-2}$ power law (Kor- 


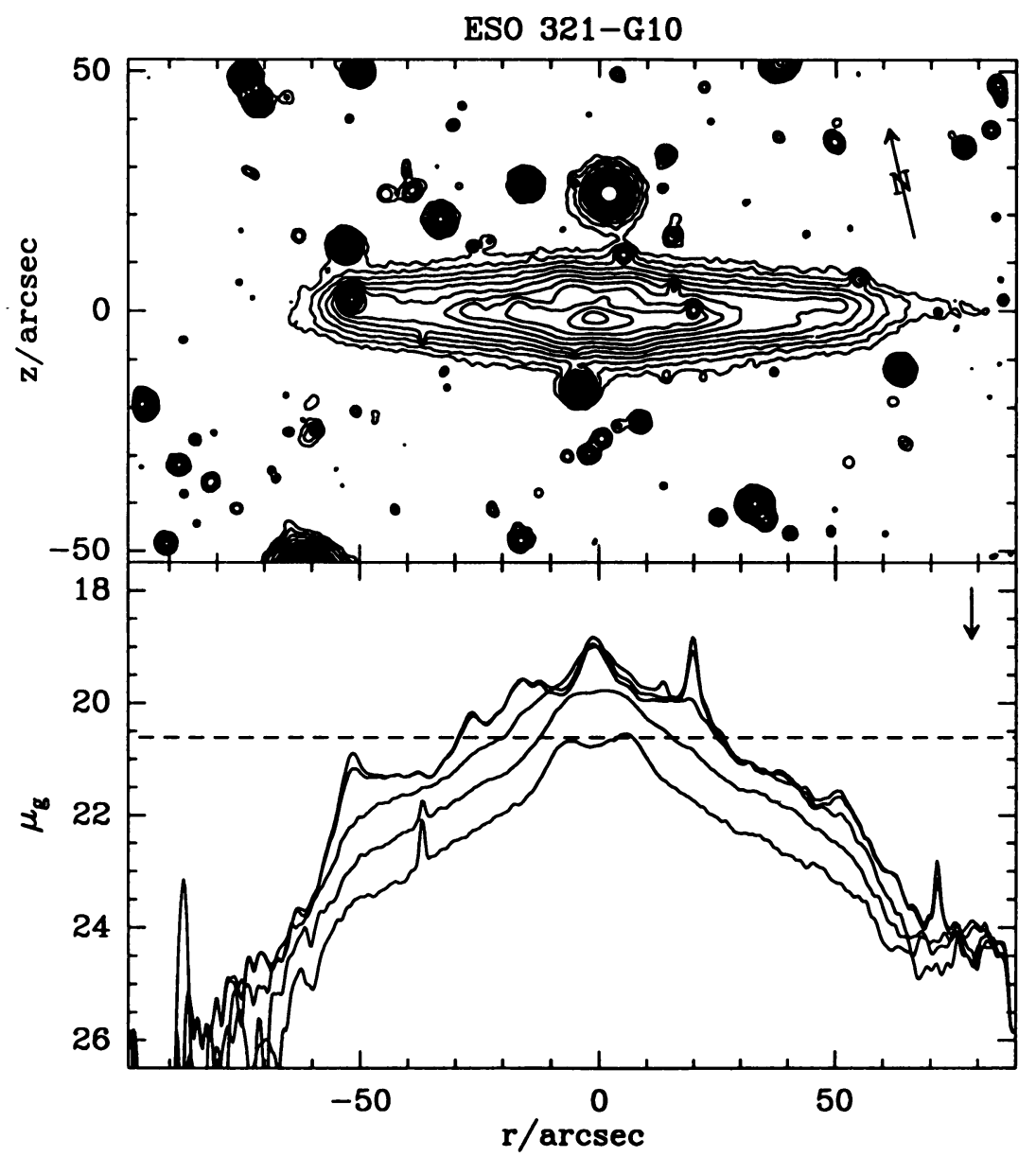

Figure 1. Top: Isophote map of the surface brightness distribution for ESO 310-G10. The faintest contour is at $\mu_{g}=24.0$, steps between contours are $0.5 \mathrm{mag}$. Bottom: Cuts parallel to the major axis separated by 1.8 arcsec; the dashed line represents the brightness of the night sky. The double humped profile identifies the $b / p$ bulge.

mendy 1982). Hamabe (1982) used a power law representation to compare spheroidal components for galaxies of morphological types ranging from $\mathrm{E}$ to Sc. The few late type cases in his diagram can be at most suggestive for a tendency showing steeper bulges in late type spirals. However, Dettmar and Wielebinski (1986) report that minor axis profiles of bulges in late type spirals, and in particular b/p bulges, are much steeper than spheroids in early type spirals and S0s. While this may support the idea of bulges in late type spirals being different from spheroids in early type spirals the result 


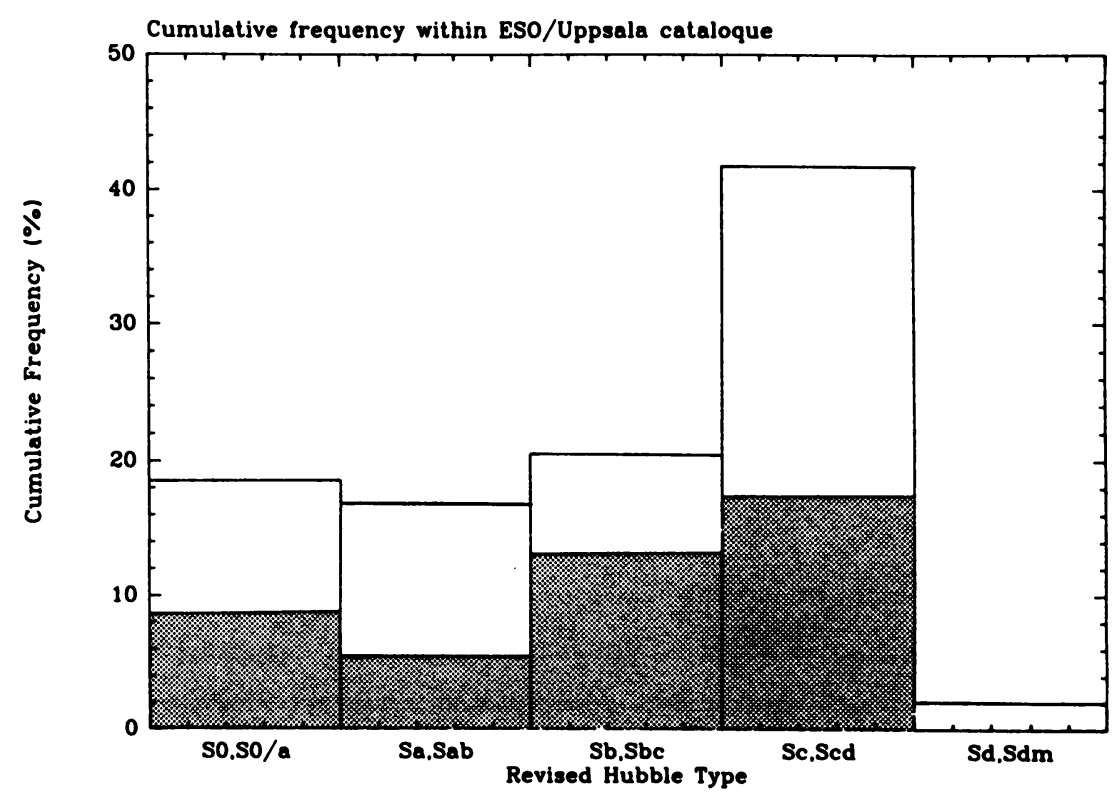

Figure 2. Cumulative frequency of morphological types (upper histogram) and b/p bulges within each bin (hatched histogram) scaled to the distribution of types in the ESO/Uppsala catalogue.

is, of cause, hampered by the influence of dust absorption. The availability of IR detector arrays will allow for a thorough reanalyses of this question in the near future.

\section{Discussion and Conclusions}

The high frequency $(>35 \%)$ of the $b / p$ bulge phenomenon requires a very common process to be at work. Various processes have been proposed: (1) external torques (May et al. 1985) , (2) bars (e.g., Combes et al. 1990), and (3) "soft" merging (Binney and Petrou 1985; Whitmore and Bell 1988). All these processes might contribute to the high frequency of $b / p$ bulges, with bars explaining the largest fraction.

This is supported by the growing number of galaxies with $b / p$ bulges that show in optical as well as radio high-resolution spectral line studies the complex gas motions characteristic for a bar. In addition to the cases mentioned before by F. Combes and K. Kuijken (this proceedings), I want to mention NGC 3268 and NGC 2683. Both objects are known for decades to show $\mathrm{b} / \mathrm{p}$ bulges. The gas kinematics in the central region obtained in $\mathrm{CO}(\mathrm{J}=2-1)$ in the case of NGC 3628 (Reuter et al. 1991) and in HI for NGC 2683 (Broeils 1992) again link the $b / p$ bulges to the presence of $a$ 
bar.

The possibility of bulges being generated by bars as an evolutionary phenomenon in disks has, of course, important implications for understanding galaxy evolution and formation. The $\mathrm{B} / \mathrm{D}$ ratio, for example, then does no longer constrain any primordial condition for galaxy formation.

Acknowledgments. I am greatful to A. Barteldrees, J. Christensen, and S. Gonzaga for help with the data reduction. Parts of this work have been supported by DFG at University of Bonn and DDRF at STScI.

\section{References}

Barteldrees, A., and Dettmar, R.-J. (1994) A\&AS 103, 475

Binney, J., and Petrou, M. (1985) MNRAS 214, 449

Broeils, A. (1992) PhD thesis, Groningen

Combes, F., Debbasch, F., Friedli, D., and Pfenniger, D. (1990) A\&A 232, 82

de Souza, R. E., and dos Anjos, S. (1987) A\&AS 70, 465

Dettmar, R.-J., and Barteldrees, A. (1990) in Bulges of Galaxies, eds. B. J. Jarvis and D. M. Terndrup, ESO, p. 255

Dettmar, R.-J., and Wielebinski, R. (1986) A\&A 167, L21

Hamabe, M. (1982) PASJ 34, 423

Jarvis, B. J. (1986) AJ 91, 65

Kormendy, J. (1982) in Morphology and Dynamics of Galaxies, eds. L. Martinet, M. Mayor, Geneva Observatory, p. 113

May, A., van Albada, T. S., and Norman, C. A. (1985) MNRAS 214, 131

Raha, N., Sellwood, J. A., James, R. A., and Kahn, F. D. (1991), Nature 352, 411

Reuter, H.-P., Krause, M., Wielebinski, R., and Lesch, H. (1991) A\&A 248, 12

Sellwood, J. A., Wilkinson, A. (1993) Rep. Prog. Phys. 56, 173

Shaw, M. A. (1987) MNRAS 229, 691

Whitmore, B. C., and Bell, M. (1988) ApJ 324, 741

Wray, J. (1988) The Colour Atlas of Galaxies, Cambridge

\section{DISCUSSION}

P. Teuben: Have any multi wavelength IR studies been done to correct for dust absorption and check how persistent these P/B bulges are? Recall Dwek showed a few days ago that in our galaxy bulge it largely disappears.

Dettmar: For the few published cases of $b / p$ bulges in edge-on galaxies studied in the IR (e.g., NGC 891) the b/p bulge is not disappearing in the IR. Bagett and Anderson have looked for the possibility to explain the $b / p$ shape by dust absorption but could rule out this. 